\title{
GRAMÍNEAS (POACEAE) DA ÁREA DE RELEVANTE INTERESSE ECOLÓGICO (ARIE) “SANTUÁRIO DE VIDA SILVESTRE DO RIACHO FUNDO”, DISTRITO FEDERAL, BRASIL ${ }^{1}$
}

\author{
Robson Rodrigues-da-Silva ${ }^{2}$ \\ Tarciso S. Filgueiras ${ }^{3}$
}

Recebido em 20/10/2001. Aceito em 08/03/2003

\begin{abstract}
RESUMO - (Gramíneas (Poaceae) da Área de Relevante Interesse Ecológico (ARIE) "Santuário de Vida Silvestre do Riacho Fundo", Distrito Federal, Brasil). Neste trabalho relata-se o levantamento florístico das espécies de Poaceae da Área de Relevante Interesse Ecológico (ARIE) "Santuário de Vida Silvestre do Riacho Fundo", Distrito Federal, Brasil. Foram encontradas 107 espécies, distribuídas em 41 gêneros, sendo 82 nativas e 25 exóticas. São apresentadas chaves analíticas para gêneros e espécies, além de ilustrações para os táxons identificados. Coelorachis aurita (Steud.) A. Camus é citada pela primeira vez para a flora do Distrito Federal.
\end{abstract}

Palavras-chave - Gramineae, lista de espécies, nova citação, florística, levantamento florístico

\begin{abstract}
Gramineae (Poaceae) of the Área de Relevante Interesse Ecológico (ARIE) "Santuário de Vida Silvestre do Riacho Fundo", Distrito Federal, Brazil). A floristic survey of the Poaceae in the Área de Relevante Interesse Ecológico (ARIE) "Santuário de Vida Silvestre do Riacho Fundo", Distrito Federal, Brazil was undertaken. Forty one genera and 107 species were found, including 82 native and 25 introduced species. Keys to genera and species and illustrations are presented. Coelorachis aurita (Steud.) A. Camus is recorded for the first time in the Distrito Federal, Brazil.
\end{abstract}

Key words - Gramineae, species list, new citation, floristics, floristic survey

\section{Introdução}

A ARIE "Santuário de Vida Silvestre do Riacho Fundo" é área de proteção permanente criada pelo governo do Distrito Federal, através do Decreto n. 1.138 de 16 de julho de 1988 para a proteção e salvaguarda da biota nativa. $\mathrm{O}$ Santuário compreende 480,12 hectares e está inserido na Área de Proteção Ambiental (APA) das bacias dos córregos Gama e Cabeça do

\footnotetext{
1 Parte da Dissertação de Mestrado do primeiro Autor

2 Jardim Botânico de Brasília, SMDB, conj. 12, Lago Sul, CEP 71620-120, Brasília, DF, Brasil (tcrobson@ hotmail.com)

3 Reserva Ecológica do IBGE, C. Postal 08770, CEP 70312-970, Brasília, DF, Brasil. Bolsista do CNPq (tfilg@uol.com.br)
} 
Veado; engloba o curso final do córrego Riacho Fundo e seu estuário, até o encontro deste com o lago Paranoá. Localiza-se entre as coordenadas $15^{\circ} 50^{\prime}-51^{\prime} \mathrm{S}$ e $47^{\circ} 56^{\prime}-57^{\prime} \mathrm{W}$.

Os solos predominantes no Santuário são o latossolo, o hidromórfico e os cambissolos, exceto às margens do Riacho Fundo, onde ocorrem solos aluviais eutróficos (FUNATURA 1994).

O clima do Distrito Federal é caracterizado pela ocorrência de duas estações bem definidas ao longo do ano. Uma estação fria e seca, de maio a setembro, e outra estação quente e úmida, de outubro a abril (SEMATEC 1992). Este clima enquadra-se na categoria Aw de Köppen, ou seja, clima tropical de Savana.

A vegetação do Santuário é composta predominantemente por Mata Ciliar e Campo Úmido, sendo também encontradas porções de Campo Limpo, Campo Sujo, Campo de Murunduns e Campo Cerrado, além de áreas com diferentes graus de perturbação ou mesmo sem a cobertura característica de Cerrado.

Este trabalho objetivou o levantamento florístico das espécies de gramíneas ocorrentes na ARIE "Santuário de Vida Silvestre do Riacho Fundo".

\section{Material e métodos}

Foram realizadas 18 viagens de coleta botânica ao Santuário, entre novembro/1997 e fevereiro/2000. O material botânico coletado foi incorporado ao acervo dos herbários da Universidade de Brasília (UB) e da Reserva Ecológica do IBGE (IBGE).

A identificação e a classificação das espécies de gramíneas foram efetuadas com base em bibliografia para a família (Chase 1942; Clayton 1969; Boldrini 1976; Filgueiras 1982; 1995; Clayton \& Renvoize 1986; Nicora \& Rúgulo de Agrasar 1987; Pohl 1980; Renvoize 1984; Stieber 1982; 1987; Sendulsky \& Soderstrom 1984; Smith \& Wasshausen 1981; Judziewicz 1990; Watson et al. 1985; Watson
\& Dalwitz 1992; Zuloaga et al. 1994; Boechat 1998; Boechat \& Longhi-Wagner 1995; LonghiWagner 1999, e outras). Dentre os materiais examinados cita-se apenas um exemplartestemunho para cada espécie (Tab. 1). Uma lista completa de todos os materiais examinados pode ser encontrada em Rodrigues-da-Silva (2000).

As ilustrações apresentadas neste trabalho têm finalidade diagnóstica ou não, tendo sido baseadas principalmente nas estruturas reprodutivas; eventualmente, características vegetativas foram ilustradas. Os desenhos foram feitos a partir dos espécimes listados na tabela 1 .

\section{Resultados e discussão}

Na ARIE "Santuário de Vida Silvestre do Riacho Fundo" foram encontradas 107 espécies de Poaceae, distribuídas em 41 gêneros, nove tribos e quatro subfamílias. Deste total, 82 são espécies nativas $(76,6 \%)$ e 25 exóticas $(23,4 \%)$; 86 espécies $(80,4 \%)$ são perenes, enquanto apenas $21(19,6 \%)$ são anuais.

A maior parte dos gêneros e espécies coletados no Santuário (32 gêneros e 88 espécies) pertence à subfamília Panicoideae. Chloridoideae (seis gêneros e 13 espécies) é a segunda subfamília em número de espécies, devido basicamente às espécies exóticas. A subfamília Arundinoideae está presente com dois gêneros e cinco espécies, sendo as espécies do gênero Aristida os seus principais representantes. A subfamília Bambusoideae não apresenta espécies nativas na área, apesar de boa parte do Santuário ser recoberta por Mata Ciliar. O único representante da subfamília (Bambusa vulgaris Schrader ex Wendl.) é espécie asiática largamente cultivada no Brasil. A ausência de espécies de bambus nativos no Santuário está provavelmente associada ao grau de antropização da Mata Ciliar.

Paspalum (23 espécies), Panicum (13 espécies), Axonopus (8 espécies), Andropogon (6 espécies) e Eragrostis (5 espécies) são os gêneros mais representativos, totalizando 51,4\% 
Tabela 1. Hábitat e material-testemunho das espécies coletadas na ARIE "Santuário de Vida Silvestre do Riacho Fundo". (A: Antrópico; Aq: Aquático; CC: Campo Cerrado; CL: Campo Limpo; CM: Campo Murundu; CS: Campo Sujo; Cum: Campo Úmido; MC: Mata Ciliar; R: Afloramento rochoso). (*) Espécie exótica.

Espécie Material-testemunho Hábitat

Acroceras zizanioides (Kunth) Dandy Agenium leptocladum (Hack) W.D. Clayton Andropogon bicornis L.

A. fastigiatus $\mathrm{Sw}$.

A. lateralis Nees

A. leucostachyus Kunth in Humb.*

A. macrothrix Trin.

A. virgatus Desv. in Hamilt.

Aristida recurvata Kunth in Humb.

A. riparia Trin.

A. setifolia Kunth in Humb.

A. torta (Nees) Kunth

Arthropogon filifolius Filg.

A. villosus Ness

Arundinella hispida (Willd.) Kuntze

Arundo donax L. *

Axonopus aureus P. Beauv.

A. barbigerus (Kunth) Hitchc.

A. brasiliensis (Spreng.) Kuhlm.

A. capillaris (Lam.) Chase

A. comans (Trin.) Kuhlm.

A. equitans Hitchc. \& Chase

A. marginatus (Trin.) Chase

A. siccus (Nees) Kuhlm.

Bambusa vulgaris Schrader ex Wendl. *

Chloris orthonoton Döll*

C. pycnothrix Trin. *

Coelorachis aurita (Steud.) A. Camus *

Cymbopogon densiflorus (Steud.) Stapf *

Cynodon dactylon (L.) Pers. *

Digitaria ciliaris (Retz.) Koel *

D. violascens Link *

Echinolaena inflexa (Poir.) Chase

Eleusine indica (L.) Gaertn. *

Eragrostis cf. acutifolia (Kunth) Nees

E. maypurensis (Kunth) Steud.

E. cf. pectinacea (Michx.) Nees *

E. pilosa (L.) P. Beauv. *

E. rufescens Schrader ex Schulter

Eriochrysis cayennensis P. Beauv.

Homolepsis glutinosa (Sw.) Zuloaga \& Soderst.

Hyparrhenia bracteata (Wild.) Stapf

H. rufa (Nees) Stapf*

Ichnanthus pallens (Sw.) Munro ex Benth.

I. procurrens (Nees ex Trin.) Swallen

I. tenuis (Presl.) Hitchc. \& Chase

Lasiacis ligulata Hitchc. \& Chase
Rodrigues-da-Silva \& D. Alvarenga 440 (UB)
Rodrigues-da-Silva 74 (UB)

Rodrigues-da-Silva 102 (UB)

Rodrigues-da-Silva 138 (UB)

Rodrigues-da-Silva 03 (UB)

Rodrigues-da-Silva (UB)

Rodrigues-da-Silva 514 (UB)

Rodrigues-da-Silva 481 (UB)

Rodrigues-da-Silva 73 (UB)

Rodrigues-da-Silva 61 (UB)

Rodrigues-da-Silva 486 (UB)

Rodrigues-da-Silva 53 (UB)

Filgueiras \& Oliveira 3207 (IBGE)

Rodrigues-da-Silva 93 (UB)

Rodrigues-da-Silva \& Alvarenga 436 (UB)

Rodrigues-da-Silva \& Alvarenga 519 (UB)

Rodrigues-da-Silva \& Alvarenga 104 (UB)

Rodrigues-da-Silva \& Alvarenga 101 (UB)

Rodrigues-da-Silva \& Alvarenga 85 (UB)

Rodrigues-da-Silva \& Alvarenga 20 (UB)

Rodrigues-da-Silva \& Alvarenga 513 (UB)

Rodrigues-da-Silva \& Alvarenga 78 (UB)

Rodrigues-da-Silva \& Alvarenga 99 (UB)

Rodrigues-da-Silva \& Alvarenga 63 (UB)

Rodrigues-da-Silva \& Alvarenga 520 (UB)

Rodrigues-da-Silva \& Alvarenga 120 (UB)

Rodrigues-da-Silva \& Alvarenga 492 (UB)

Rodrigues-da-Silva \& Alvarenga 125 (UB)

Rodrigues-da-Silva \& Alvarenga 113 (UB)

Rodrigues-da-Silva \& Alvarenga 152 (UB)

Rodrigues-da-Silva \& Alvarenga 458 (UB)

Rodrigues-da-Silva \& Oliveira 449 (UB)

Rodrigues-da-Silva \& Oliveira 103 (UB)

Rodrigues-da-Silva \& Oliveira 153 (UB)

Rodrigues-da-Silva \& Oliveira 460 (UB)

Rodrigues-da-Silva \& Oliveira 512 (UB)

Rodrigues-da-Silva \& Oliveira 459 (UB)

Rodrigues-da-Silva \& Oliveira 493 (UB)

Rodrigues-da-Silva \& Oliveira 130 (UB)

Rodrigues-da-Silva \& Oliveira 44 (UB)

Rodrigues-da-Silva \& Alvarenga 433 (UB)

Rodrigues-da-Silva \& Oliveira 468 (UB)

Rodrigues-da-Silva \& Oliveira 67 (UB)

Rodrigues-da-Silva \& Oliveira 461 (UB)

Rodrigues-da-Silva \& Oliveira 139 (UB)

Rodrigues-da-Silva \& Oliveira 465 (UB)

Rodrigues-da-Silva \& Oliveira 487 (UB)
$\mathrm{MC}$

CL

Cum, A

A

Cum, CL, CS, CM

$\mathrm{CL}, \mathrm{CM}$

Cum, CM, CS

$\mathrm{CM}, \mathrm{Cum}$

$\mathrm{CS}, \mathrm{CC}$

$\mathrm{CL}, \mathrm{CS}$

CS, CC, Cum, A

$\mathrm{CC}$

Cum

$\mathrm{CM}$, Cum

MC,Cum

A

$\mathrm{CC}, \mathrm{CM}$

Cum, CC, A

Cum

CL

Cum

CL

$\mathrm{CM}$

CC

A

A

A

A

A

A

A

A

CC, CL

A

MC

A

A

A

Cum

Cum, CM

$\mathrm{MC}$

Cum

A

MC

$\mathrm{CM}$

$\mathrm{MC}$

MC 
Tabela 1 (continuação)

Espécie Material-testemunho Hábitat

Leptocoryphium lanatum (Kunth) Nees

Melinis minutiflora P. Beauv. *

Mesosetum ferrugineum (Trin.) Chase

Microchloa indica (L. f.) P. Beauv. *

Oplismenus hirtellus (L.) P. Beauv.

Otachyrium seminudum Hack. ex Send. $\&$ Soderstr.

O. versicolor (Döll) Henr.

Panicum caaguazuense Henr.

P. cyanescens Trin.

P. dichotomiflorum Michx.

P. laxum Sw.

P. maximum Jacq. *

P. olyroides Kunth

P. parvifolium Lam.

P. peladoense Henr.

P. pilosum $\mathrm{Sw}$.

P. pseudisachne Mez

P. schwackeanum $\mathrm{Mez}$

P. sellowii Nees

$P$ trichanthum Nees

Paspalum carinatum Humb. \& Bonpl.

P. clavuliferumWright

P. conjugatum Berg. *

P. conspersum Schrad.

P. dedeccae Quarín

P. ellipticum Döll

$P$. erianthum Nees ex Trin.

P. fasciculatum Willd. ex Flügge

$P$. gardnerianum Nees

P. gemminiflorum Steud.

P. glaucescens Hack.

$P$. hyalinum Nees

P. imbricatum Filg.

P. maculosum Trin.

P. multicaule Poir.

P. paniculatum $\mathrm{L}$.

P. pectinatum Nees

P. pilosum Lam. *

P. plicatulum Michx.

P. polyphyllum Nees

$P$. reduncum Nees ex Steud.

P. stellatum Humb. \& Bonpl.

P. trachycoleon Steud.

Pennisetum polystachyon (L.) Schult. *

P. purpureum Schum. *

Rhynchelytrum repens (Willd.) C. E. Hubb. *

Saccharum trinii Steud.

Schizachyrium microstachyum (Desv.) Roseng.

$S$. cf. sanguineum (Retz.) Alst.
Rodrigues-da-Silva \& Oliveira 96 (UB)

Rodrigues-da-Silva \& Oliveira 471 (UB)

Rodrigues-da-Silva \& Oliveira 89 (UB)

Rodrigues-da-Silva \& Oliveira 19 (UB)

Rodrigues-da-Silva \& Oliveira 491 (UB)

Rodrigues-da-Silva \& Oliveira 146 (UB)

Rodrigues-da-Silva \& Oliveira 28 (UB)

Rodrigues-da-Silva 504 (UB)

Rodrigues-da-Silva 46 (UB)

Rodrigues-da-Silva 129 (UB)

Rodrigues-da-Silva 111 (UB)

Rodrigues-da-Silva \& Alvarenga 437 (UB)

Rodrigues-da-Silva \& Alvarenga 57 (UB)

Rodrigues-da-Silva \& Oliveira 455 (UB)

Rodrigues-da-Silva \& Oliveira 68 (UB)

Rodrigues-da-Silva \& Alvarenga 439 (UB)

Rodrigues-da-Silva \& Alvarenga 521 (UB)

Rodrigues-da-Silva \& Alvarenga 128 (UB)

Rodrigues-da-Silva \& Alvarenga 488 (UB)

Rodrigues-da-Silva \& Alvarenga 475 (UB)

Rodrigues-da-Silva \& Alvarenga 48 (UB)

Rodrigues-da-Silva \& Alvarenga 506 (UB)

Rodrigues-da-Silva \& Alvarenga 441 (UB)

Rodrigues-da-Silva \& Alvarenga 435 (UB)

Rodrigues-da-Silva \& Alvarenga 91 (UB)

Rodrigues-da-Silva \& Alvarenga 90-A (UB)

Rodrigues-da-Silva \& Alvarenga 95 (UB)

Rodrigues-da-Silva \& Alvarenga 442 (UB)

Rodrigues-da-Silva \& Alvarenga 50 (UB)

Rodrigues-da-Silva \& Alvarenga 64 (UB)

Rodrigues-da-Silva \& Alvarenga 37 (UB)

Rodrigues-da-Silva \& Alvarenga 522 (UB)

Rodrigues-da-Silva \& Alvarenga 36 (UB)

Rodrigues-da-Silva \& Alvarenga 13 (UB)

Rodrigues-da-Silva \& Alvarenga 505 (UB)

Rodrigues-da-Silva \& Alvarenga 103 (UB)

Rodrigues-da-Silva \& Alvarenga 88 (UB)

Rodrigues-da-Silva \& Oliveira 454 (UB)

Rodrigues-da-Silva \& Oliveira 51 (UB)

Rodrigues-da-Silva \& Oliveira 81 (UB)

Rodrigues-da-Silva \& Oliveira 76 (UB)

Rodrigues-da-Silva \& Oliveira 70 (UB)

Rodrigues-da-Silva \& Oliveira 65 (UB)

Rodrigues-da-Silva \& Oliveira 515 (UB)

Rodrigues-da-Silva \& Alvarenga 438 (UB)

Rodrigues-da-Silva \& Alvarenga 80 (UB)

Rodrigues-da-Silva \& Alvarenga 24 (UB)

Rodrigues-da-Silva \& Alvarenga 516 (UB)

Rodrigues-da-Silva \& Alvarenga 497 (UB)
CM, Cum

A, Cum

$\mathrm{CM}$

A

$\mathrm{MC}$

Cum, MC

Cum

Cum

Cum

Aq, Cum

$\mathrm{MC}$

A

$\mathrm{CC}, \mathrm{CL}, \mathrm{CS}$

Cum

CL

$\mathrm{MC}$

Cum

Cum

$\mathrm{MC}$

$\mathrm{MC}$

CS

A

A

MC

Cum

$\mathrm{CM}$

CM

$\mathrm{MC}$, Cum

CC, CL,CS

$\mathrm{CC}, \mathrm{CS}$

$\mathrm{CM}, \mathrm{MC}$

$\mathrm{R}$

Cum, CM

Cum, CS

A

MC, Cum

$\mathrm{CM}$

A

CC

CL

$\mathrm{CL}, \mathrm{CC}, \mathrm{CM}$

CL

CL

A

A

A

Cum

CS

$\mathrm{CM}$ 
Tabela 1 (continuação)

Espécie

Material-testemunho

Hábitat

S. tenerum Nees

Setaria parviflora (Poir.) Kerguélen *

Sorghastrum minarum (Nees) Hitchc.

Sporobolus acuminatus (Trin.) Hack.

S. ciliatus Presl

S. indicus (L.) R. Br. *

Steinchisma decipiens (Nees ex Trin.) W. V. Br.

S. hians (Elliott) Nash

Thrasya petrosa (Trin.) Chase

Trachypogon spicatus (L. f.) Kuntze

Urochloa brizantha (Hochst. ex A. Rich.)

Webster *
Rodrigues-da-Silva \& Alvarenga 502 (UB)

Rodrigues-da-Silva \& Alvarenga 446 (UB)

Rodrigues-da-Silva \& Alvarenga 141 (UB)

Rodrigues-da-Silva \& Alvarenga 62 (UB)

Rodrigues-da-Silva \& Alvarenga 510 (UB)

Rodrigues-da-Silva \& Alvarenga 494 (UB)

Rodrigues-da-Silva \& Alvarenga 150 (UB)

Rodrigues-da-Silva \& Oliveira 453 (UB)

Rodrigues-da-Silva \& Oliveira 112 (UB)

Rodrigues-da-Silva \& Oliveira 72 (UB)

Rodrigues-da-Silva \& Oliveira 508 (UB)
CL, CM, Cum

A

Cum

CS, CC

$\mathrm{CM}, \mathrm{A}$

A

Cum

$\mathrm{MC}$

$\mathrm{CS}, \mathrm{CC}$

$\mathrm{CS}$

A das espécies. Vinte e cinco gêneros estão representados por apenas uma única espécie, ou seja $23,4 \%$ das espécies encontradas no Santuário.

As 82 espécies de gramíneas nativas encontradas no Santuário representam 5,6\% das espécies brasileiras (Burman 1985), 21,6\% das espécies confirmadas para o Cerrado (Mendonça et al. 1998) e 38,8\% das espécies ocorrentes no Distrito Federal (Filgueiras 1991).

Entre os habitats encontrados no Santuário, Campo Úmido e as Áreas Antrópicas (com variado grau de perturbação) apresentaram o maior número de espécies (34 e 32 respectivamente), seguidos por Campo Murundu (19), Mata Ciliar (18), Campo Limpo (15), Campo Sujo (14) e Campo Cerrado (14). Panicum dichotomiflorum Michx., considerada espécie aquática, foi coletada dentro e fora d'água. Paspalum hyalinum Nees foi coletada em pequeno afloramento rochoso.

Dentre os registros de gramíneas coletadas no Santuário, antes da realização deste trabalho, apenas Arthropogon filifolius Filg. (Filgueiras
\& Oliveira 3207 IBGE) não foi por nós coletado. Coelorachis aurita (Steud.) A. Camus é nova citação para a flora do Distrito Federal.

Dentre as espécies nativas encontradas, 57 possuem algum valor forrageiro, segundo os critérios adotados por Filgueiras (1992), sendo que 10 delas são consideradas de alto valor forrageiro (Filgueiras l.c.).

Há necessidade de se estabelecer um programa de reabilitação ecológica das áreas antrópicas do Santuário, usando-se inclusive gramíneas nativas (Martins 1996). É também urgente o estabelecimento de plano de manejo das populações residentes de capivara (Hydrochaeris hydrochaeris) no Santuário. Como estes roedores encontram-se em grande número no local, suas populações estão provavelmente alterando a composição da flora graminóide. O sobrepastejo das espécies mais palatáveis pode levar algumas delas à extinção no Santuário.

A seguir, são apresentadas chaves de identificação para os gêneros e espécies de ocorrência confirmada no local. 
Chave para os gêneros de gramíneas da ARIE

"Santuário de Vida Silvestre do Riacho Fundo"

(Quando o gênero está representado por uma única espécie, esta aparece diretamente nesta chave)

1. Colmos 5-15m compr. e 3-8cm diâm.; folhas com pseudopecíolo (Fig. 5-R-S-T)

Bambusa vulgaris

1. Colmos com, no máximo, 3,5m compr. e 2,5cm diâm.; folhas sem pseudopecíolo

2. Lemas densamente pilosos (Fig. 1-C-D)

Arundo donax

2. Lemas glabros a glabrescentes

3. Espiguetas aos pares, uma séssil e a outra pedicelada

4. Inflorescência em racemos paucifloros, vilosos, cilíndricos a subcilíndricos; raque frágil

5. Inflorescência subcilíndrica; espiguetas revestidas por pilosidade branca (Fig. 2-Q)

Saccharum trinii

5. Inflorescência cilíndrica; espiguetas revestidas por pilosidade marrom a dourada (Fig. 2-R)

Eriochrysis cayennensis

4. Inflorescência em racemos isolados, pareados, alternos ou digitados, nunca cilíndrica ou subcilíndrica; raque rígida

6. Racemos com um a vários pares de espiguetas basais isomorfas e múticas, distintos dos pares superiores heteromorfos

7. Espiguetas glabras (Fig. 2-E-F)

Agenium leptocladum

7. Espiguetas revestidas por pilosidade marrom a ferrugínea Hyparrhenia

6. Racemos com todos os pares de espiguetas isomorfos, espiguetas basais sem distinção das demais

8. Espigueta pedicelada com aurícula conspícua no pedicelo (Fig. 5-Q) Coelorachis aurita

8. Espigueta pedicelada com pedicelo sem aurículas

9. Espigueta pedicelada hermafrodita (Fig. 2-A) Trachypogon spicatus

9. Espigueta pedicelada masculina ou neutra

10. Plantas com folhas aromáticas Cymbopogon densiflorus

10. Plantas com folhas não aromáticas

11. Espigueta pedicelada ausente e representada apenas por seu pedicelo; inflorescência dourada (Fig. 2-L)

Sorghastrum minarum

11. Espigueta pedicelada presente; inflorescência nunca dourada

12. Espigueta séssil com a gluma inferior sulcada ou côncava

Andropogon

12. Espigueta séssil com a gluma inferior plana ou convexa Schizachyrium

3. Espiguetas solitárias ou em fascículos, se aos pares, ambas pediceladas

13. Espiguetas subtendidas por uma ou mais cerdas

14. Cerdas caducas com as espiguetas Pennisetum

14. Cerdas persistentes na raque (Fig. 2-K) Setaria parviflora

13. Espiguetas livres, sem cerdas 
15. Espiguetas uniflosculadas

16. Inflorescência em racemos ou em espiga

17. Inflorescência em espiga solitária, arqueada (Fig. 5-O)

Microchloa indica

17. Inflorescência em racemos digitados ou subdigitados (Fig. 5-U) Cynodon dactylon

16. Inflorescência em panícula

18. Lema com três aristas.... Aristida

18. Lema mútico Sporobolus

15. Espiguetas com dois ou mais flósculos

19. Espiguetas com três ou mais flósculos

20. Inflorescência em racemos digitados ou subdigitados (Fig. $1-\mathrm{M})$ Eleusine indica

20. Inflorescência em panícula laxa a espiciforme Eragrostis

19. Espiguetas biflosculadas

21. Inflorescência em racemos digitados ou subdigitados Chloris

21. Inflorescência em panícula

22. Flósculo superior com o lema e a pálea mais delicados do que as glumas

23. Espiguetas lateralmente comprimidas; aristas retorcidas e geniculadas (Fig. 1-A) .... Arundinella hispida

23. Espiguetas cilíndricas; aristas retas Arthropogon

22. Flósculo superior com o lema e a pálea mais firmes do que as glumas

24. Inflorescência em racemos

25. Inflorescência em racemo solitário

26. Raque alada; espiguetas dispostas face a face (dorso com dorso) (Fig. 3-X)

Thrasya petrosa

26. Raque não alada; espiguetas nunca dispostas face a face (dorso com dorso)

27. Racemos apresentando uma torção de aproximadamente $90^{\circ} \mathrm{em}$ relação ao pedúnculo; espigueta terminal simulando uma extensão da raque (Fig. 5-I-i) ........ Echinolaena inflexa

27. Racemos retos; espiguetas dorsalmente comprimidas (Fig. 5M-m) Mesosetum ferrugineum

25. Inflorescência em dois ou mais racemos

28. Gluma inferior aristada (Fig. 2-O) Oplismenus hirtellus

28. Gluma inferior ausente ou reduzida, raro mútica ou mucronada 
29. Lema superior com o dorso cartilaginoso e as margens membranáceas

Digitaria

29. Lema superior totalmente cartilaginoso

30. Gluma inferior sempre presente, com $1 / 2$ a $3 / 4$

do comprimento da espigueta (Fig.5-L-1)

Urochloa brizantha

30. Gluma inferior ausente ou reduzida

31. Lema superior com o dorso próximo à raque

Paspalum

31. Lema superior com o dorso distante da raque

Axonopus

24. Inflorescência em panícula

32. Lema superior com par de apêndices ou cicatrizes basais

Ichnanthus

32. Lema superior sem apêndices ou cicatrizes basais

33. Flósculo superior exposto

Otachyrium

33. Flósculo superior nunca exposto

34. Flósculo superior apiculado (Fig. 3-S-s) ....Acroceras zizanioides

34. Flósculo superior nunca apiculado

35. Espiguetas viscosas na maturidade (Fig. 5-P-p)

Homopelis glutinosa

35. Espiguetas nunca viscosas na maturidade

36. Gluma superior aristada

37. Espiguetas glabras; lâminas foliares recobertas por pêlos secretores (Fig. 2-N)

Melinis minutiflora

37. Espiguetas densamente pilosas, pêlos róseos, tornando-se brancos com a maturidade; folhas sem pêlos secretores (Fig. 2-U)

Rhynchelytrum repens

36. Gluma superior mútica

38. Espiguetas recobertas por pêlos lanosos (Fig. 3V-v)

Leptocoryphyum lanatum

38. Espiguetas glabras ou com pêlos curtos

39. Espiguetas subglobosas, obliquamente inseridas no pedicelo (Fig. 5-N-n)

Lasiacis ligulata

39. Espiguetas geralmente achatadas dorso-ventralmente, inseridas eretas no pedicelo

40. Pálea inferior expandida na maturidade Steichisma

40. Pálea inferior não expandida na maturidade 
Chaves para as espécies de ocorrência confirmada na ARIE

"Santuário de Vida Silvestre do Riacho Fundo"

\section{Andropogon L.}

1. Plantas anuais (Fig. 2-I)

A. fastigiatus

1. Plantas perenes

2. Inflorescência em panícula de racemos complexamente ramificados

3. Espigueta séssil mútica (Fig. 2-G)

A. bicornis

3. Espigueta séssil aristada (Fig. 2-H)

A. virgatus

2. Inflorescência em racemos digitados ou subdigitados

4. Espiguetas séssil e pedicelada subiguais (Fig. 2-B)

A. lateralis

4. Espigueta pedicelada reduzida, atingindo no máximo $1 / 2$ do comprimento da espigueta séssil

5. Espigueta séssil com arista retorcida (Fig. 2-C)

A. macrothrix

5. Espigueta séssil mútica (Fig. 2-D)

A. leucostachyus

\section{Aristida L.}

1. Colmos ramificados na base; arista com articulação no ápice da coluna (Fig. 1-B) ...... A. setifolia

1. Colmos não ramificados; arista sem articulação na coluna

2. Aristas sem coluna, arista central curva ou flexuosa, maior que as aristas laterais

(Fig. 1-L)

A. torta

2. Arista com coluna, arista central e laterais subiguais

3. Flósculo com calo bífido; aristas 28-55mm compr., planas (Fig. 1-G)

A. riparia

3. Flósculo com calo inteiro; aristas $8-20 \mathrm{~mm}$ compr., retorcidas e entrecruzadas na base (Fig. 1-E)

A. recurvata

\section{Arthropogon Nees}

1. Lâmina foliar com até $2 \mathrm{~mm}$ larg.; espiguetas glabras na base ou com pêlos curtos, menores de $2 \mathrm{~mm}$ compr. (Fig. 1-F)

A. filifolius

1. Lâmina foliar com mais de $4 \mathrm{~mm}$ larg.; pêlos na base da espigueta com mais de 2mm compr. (Fig. 1-O)

A. villosus

Axonopus P. Beauv.

1. Plantas anuais (Fig. 5-E-e)

A. capillaris

1. Plantas perenes

2. Inflorescência formada por até 6 racemos

3. Espiguetas revestidas por pêlos claros de base tubercular (Fig. 5-B-b) .... A. brasiliensis

3. Espiguetas glabras ou revestidas de pêlos claros sem base tubercular ou com pêlos coloridos

4. Racemos com a raque glabra, espiguetas glabras ou glabrescentes, pêlos sempre claros

5. Plantas densamente cespitosas, formando grandes touceiras; lâminas filiformes, 20-50×0,1 cm (Fig. 5-F-f) 
5. Plantas modestamente cespitosas; lâminas conduplicadas, 11-20×0,20,4cm (Fig. 5-D-d)

A. equitans

4. Racemos e espiguetas pilosas, pêlos claros ou coloridos

6. Raque e espiguetas revestidas por pêlos dourados (Fig. 5-A)

A. aureus

6. Raque glabra, espiguetas com pêlos púrpura ou roxos margeando as nervuras (Fig. 5-H-h)

A. marginatus

2. Inflorescência formada por 8 a inúmeros racemos

7. Lâminas foliares largas, 20-55×0,3-0,6cm; lígula pilosa (Fig. 5-C-c) ..... A. barbigerus

7. Lâminas foliares filiformes, 10-45×0,1-0,3cm; lígula membrano-ciliada (Fig. 5-G-g) A. siccus

\section{Chloris Sw.}

1. Plantas perenes; flósculo superior 1,5-2mm compr. (Fig. 5-K) C. orthonoton

1. Plantas anuais; flósculo superior reduzido, ca. 1mm compr. (Fig. 5-J) C. pycnothrix

\section{Digitaria Haller}

1. Espiguetas aos pares, 3-3,3mm compr. (Fig. 4-W-w) ... D. ciliaris

1. Espiguetas em tríades, 1,4-1,8mm compr. (Fig. 4-X-x)

D. violascens

\section{Eragrostis N. M. Wolf}

1. Inflorescência com ramos basais verticilados..... E. pilosa

1. Inflorescência com ramos basais não verticilados

2. Lemas com ápices curvos para fora E. maypurensis

2. Lemas com ápices retos

3. Plantas perenes

E. cf. acutifolia

3. Plantas anuais

4. Gluma inferior linear a estreitamente lanceolada

E. rufescens

4. Gluma inferior oval a largamente lanceolada E. cf. pectinacea

Hyparrhenia Anders. ex P. Fourn.

1. Racemos 1-1,5cm compr.; espigueta séssil com arista medindo 10-20mm compr.; plantas restritas a ambientes úmidos (Fig. 2-T)

H. bracteata

1. Racemos 2-5cm compr.; espigueta séssil com arista de 20-25mm compr.; plantas ruderais (Fig. 2-S) H. rufa

Ichnanthus P. Beauv.

1. Plantas eretas; espiguetas congestas na porção mediana dos ramos, exceto uma espigueta terminal (Fig. 3-R)

I. procurrens

1. Plantas estoloníferas; espiguetas nunca congestas.

2. Plantas anuais (Fig. 3-Q)

2. Plantas perenes (Fig. 3-P) 


\section{Otachyrium Nees}

1. Inflorescência laxa a semi-contraída (Fig. 3-T) ............................................. O. versicolor

1. Inflorescência contraída (Fig. 3-U) ........................................................... O. seminudum

\section{Panicum L.}

1. Inflorescência em racemos, lígula ausente (Fig. 3-N-n)

P. pilosum

1. Inflorescência em panícula, lígula presente

2. Ramos terciários da inflorescência geralmente curtos e paucifloros, dando aos ramos secundários aspecto de racemos (Fig. 3-L-1)

P. laxum

2. Ramos terciários evidentes

3. Panícula com os ramos basais verticilados e os superiores alternos; flósculo superior rugoso (Fig. 3-K-k)

P. maximum

3. Ramos da panícula alternos; flósculo superior liso, raro finamente rugoso

4. Lema do flósculo superior com tufo de tricomas laterais na base (Fig.

3-O-O)

P. olyroides

4. Lema do flósculo superior sem tricomas na base

5. Flósculo superior castanho-escuro na maturidade

6. Plantas estoloníferas; espiguetas medindo 1,9-2,1 mm compr.; gluma inferior 1-nervada (Fig. 3-B-b)

P. sellowii

6. Plantas cespitosas; espiguetas com 2,8-3,2mm compr.; gluma inferior 5-nervada (Fig. 3-E-e)

P. peladoense

5. Flósculo superior verde a estramíneo na maturidade

7. Plantas anuais; colmos esponjosos; folhas e espiguetas geralmente com máculas violáceas (Fig. 3-D-d)

$\boldsymbol{P}$. dichotomiflorum

7. Plantas perenes; colmos nunca esponjosos; folhas e espiguetas sem máculas violáceas

8. Lâmina foliar com as nervuras paralelas à nervura central evidentes; gluma inferior enérvia (Fig. 3-I-i)

P. tricanthum

8. Lâmina foliar apenas com a nervura central evidente; gluma inferior 1-3 nervada

9. Panícula com $12-25 \times 11-25 \mathrm{~cm}$; espiguetas terminais em pedicelos longos de, no mínimo, 15mm compr. (Fig. 3-H-h)

P. caaguazuense

9. Panícula com 4-12×2-10cm; pedicelos curtos de no máximo $8 \mathrm{~mm}$ compr.

10. Espiguetas verdes; lâminas foliares medindo, no máximo; 3,3cm compr. (Fig. 3-M-m) P. parvifolium

10. Espiguetas roxas; lâminas foliares medindo 2,5 a $7,5 \mathrm{~cm}$ compr.

11. Espiguetas pilosas (Fig. 3-J-j)

P. pseudisachne

11. Espiguetas glabras

12. Espiguetas com 1,1-1,4 mm compr. (Fig. 3-A-a) P. schwackeanum

12. Espiguetas com 1,5-1,8 mm compr. (Fig. $3-\mathrm{C}-\mathrm{c})$ P. cyanescens 


\section{Paspalum L.}

1. Plantas anuais

2. Espiguetas revestidas com pêlos capitados

3. Racemos aos pares (Fig. 4-Q-q) P. multicaule

3. Racemos solitários (Fig. 4-R-r) P. clavuliferum

2. Espiguetas glabras ou glabrescentes, sem pêlos capitados

4. Inflorescência formada por 2-8 racemos alternos com o par terminal eventualmente conjugado; espiguetas medindo 1-1,3mm compr. (Fig. 4-O-o)

P. hyalinum

4. Inflorescência em racemos solitários; espiguetas medindo 2,5-3mm compr. (Fig. 4-V-v)

P. pilosum

1. Plantas perenes

5. Racemos solitários ou aos pares, conjugados ou subconjugados

6. Raque alada; racemos conjugados ou solitários; espiguetas pilosas (Fig. 4-A)

P. stellatum

6. Raque não alada; racemos conjugados ou subconjugados; espiguetas glabras ou glabrescentes

7. Plantas cespitosas; lâmina foliar $15-40 \mathrm{~cm}$ compr.; espiguetas $2,2-2,8 \mathrm{~mm}$ compr., com manchas (máculas) na gluma superior e no lema inferior (Fig. 4-K-k)

P. maculosum

7. Plantas estoloníferas; lâmina foliar 4-10cm compr.; espiguetas $1,8-2 \mathrm{~mm}$ compr., sem manchas (máculas) na gluma superior e no lema inferior (Fig. 4-N-n)

P. conjugatum

5. Três a vários racemos alternos

8. Glumas inferior e superior ausentes

9. Espiguetas medindo 4-5mm compr. (Fig. 4-S-s)

P. dedeccae

9. Espiguetas com 1,8-2,5mm compr.

10. Racemos com, no máximo, $3 \mathrm{~cm}$ compr., recurvados na maturidade; raque 1,8-2,5mm larg.; espiguetas 1,2-1,8mm compr. (Fig. 4-C-cD)

P. reduncum

10. Racemos $2,5-5 \mathrm{~cm}$ compr., eretos na maturidade; raque $0,5-1 \mathrm{~mm}$ larg.; espiguetas 1,9-2,2mm compr.(Fig. 4-E-e)

P. gardnerianum

8. Gluma superior sempre presente

11. Raque alada, $2-4,5 \mathrm{~mm}$ larg.

12. Espiguetas 2-4,5mm compr.; gluma superior e lema inferior de base inteira

13. Lâminas planas, lanceoladas; 4-6 racemos medindo $2,5-5 \mathrm{~cm}$ compr.; raque 3,8-4,3 mm larg., totalmente roxa (Fig. 4-G-g)

P. trachycoleon

13. Lâminas revolutas, aciculares; $1-2$ racemos medindo $5,5-13 \mathrm{~cm}$ compr.; raque 2,5-3,8mm larg. (Fig. 4-F-f)

P. carinatum

12. Espiguetas 4,5-8,2mm compr.; gluma superior e lema inferior de base cordada 
14. Racemos 2-3, par terminal conjugado; espiguetas 7-8,5mm compr.; margens do lema inferior revestidas com pêlos de base tubercular (Fig. 4-Y-y)

P. pectinatum

14. Racemos 5-8, alternos; espiguetas 4,5-6mm compr.; lema inferior glabro (Fig. 4-Z-z)

P. imbricatum

11. Raque não alada, com no máximo, 1,7mm larg.

15. Flósculo superior castanho-escuro

16. Lâmina foliar 0,9-2,2cm larg.; racemos 12-25; flósculo superior rugoso (Fig. 4-I-i)

P. conspersum

16. Lâmina foliar $0,3-0,6 \mathrm{~cm}$ larg.; racemos 1-7; flósculo superior liso ou suavemente papiloso

17. Gluma superior e lema inferior com rugosidade dourada (Fig. 4-L-1) P. geminiflorum

17. Gluma superior e lema inferior sem rugosidade

18. Lígula conspícua (Fig. 4-T-t)

P. glaucescens

18. Lígula inconspícua (Fig. 4-P-p)

P. plicatulum

15. Flósculo superior verde a estramíneo

19. Espiguetas glabras a pubescentes

20. Colmos 130-180cm compr.; espiguetas 2,8-3mm compr.

(Fig. 4-M-m)

P. fasciculatum

20. Colmos 16-70cm compr.; espiguetas 1,1-1,5mm compr.

(Fig. 4-J-j)

P. paniculatum

19. Espiguetas conspicuamente pilosas

21. Lâmina foliar 3-5,5cm compr.; espiguetas 2,2-2,8mm compr. (Fig. 4-B-b)

P. polyphyllum

21. Lâmina foliar 7-65 cm compr.; espiguetas 3,2-4,5mm compr.

22. Lâminas conduplicadas, 0,2-0,3mm larg.; espiguetas oblongo-elípticas, com pilosidade lanosa restrita às margens; flósculo superior rugoso (Fig. 4-U-u) ........P. ellipticum

22. Lâminas planas, 0,5-1,5mm larg.; espiguetas elípticolanceoladas totalmente revestidas por pêlos lanosos; flósculo superior papiloso (Fig. 4-H-h)

P. erianthum

\section{Pennisetum Rich.}

1. Plantas com 0,7-1,5m compr.; colmos herbáceos; inflorescências terminais e laterais; uma espigueta por fascículo (Fig. 2-M) P. polystachyum

1. Plantas com 1,2-3,5m compr.; colmos basais lignificados; inflorescências terminais; três espiguetas por fascículo (Fig. 2-P).

P. purpureum

\section{Schizachyrium Nees}

1. Inflorescência flabelada, panícula de racemos (Fig. 2-V) S. microstachyum

1. Inflorescência nunca flabelada

2. Colmos 25-70cm compr., verdes a estramíneos (Fig. 2-X) S. tenerum

2. Colmos 70-125cm compr., avermelhados (Fig. 2-Y) S. cf. sanguineum 


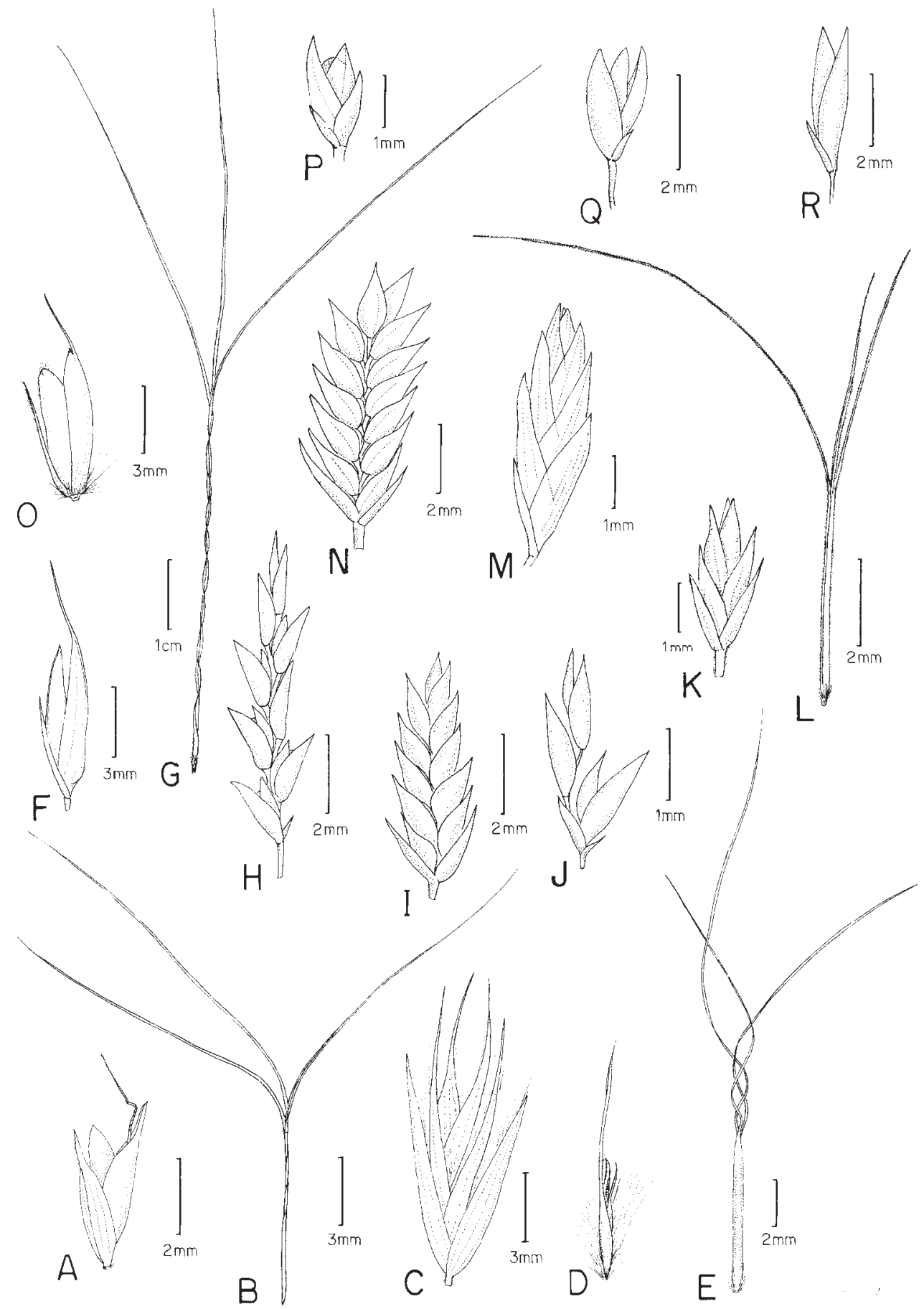

Figura 1. A - Arundinella hispida, espigueta; B - Aristida setifolia, flósculo; C - Arundo donax, espigueta; D - flósculo; E - Aristida recurvata, flósculo; F - Arthropogon filifolius, espigueta; G - Aristida riparia, flósculo; H - Eragrostis pilosa, espigueta; I - E. rufescens, espigueta; J - E. cf. pectinacea, espigueta; K - E. cf. acutifolia, espigueta; L Aristida torta, flósculo; M - Eleusine indica, espigueta; N - Eragrostis maypurensis, espigueta; O - Arthropogon villosus, espigueta; P - Sporobolus indicus, espigueta; Q - S. ciliatus, espigueta; R - S. acuminatus, espigueta. 


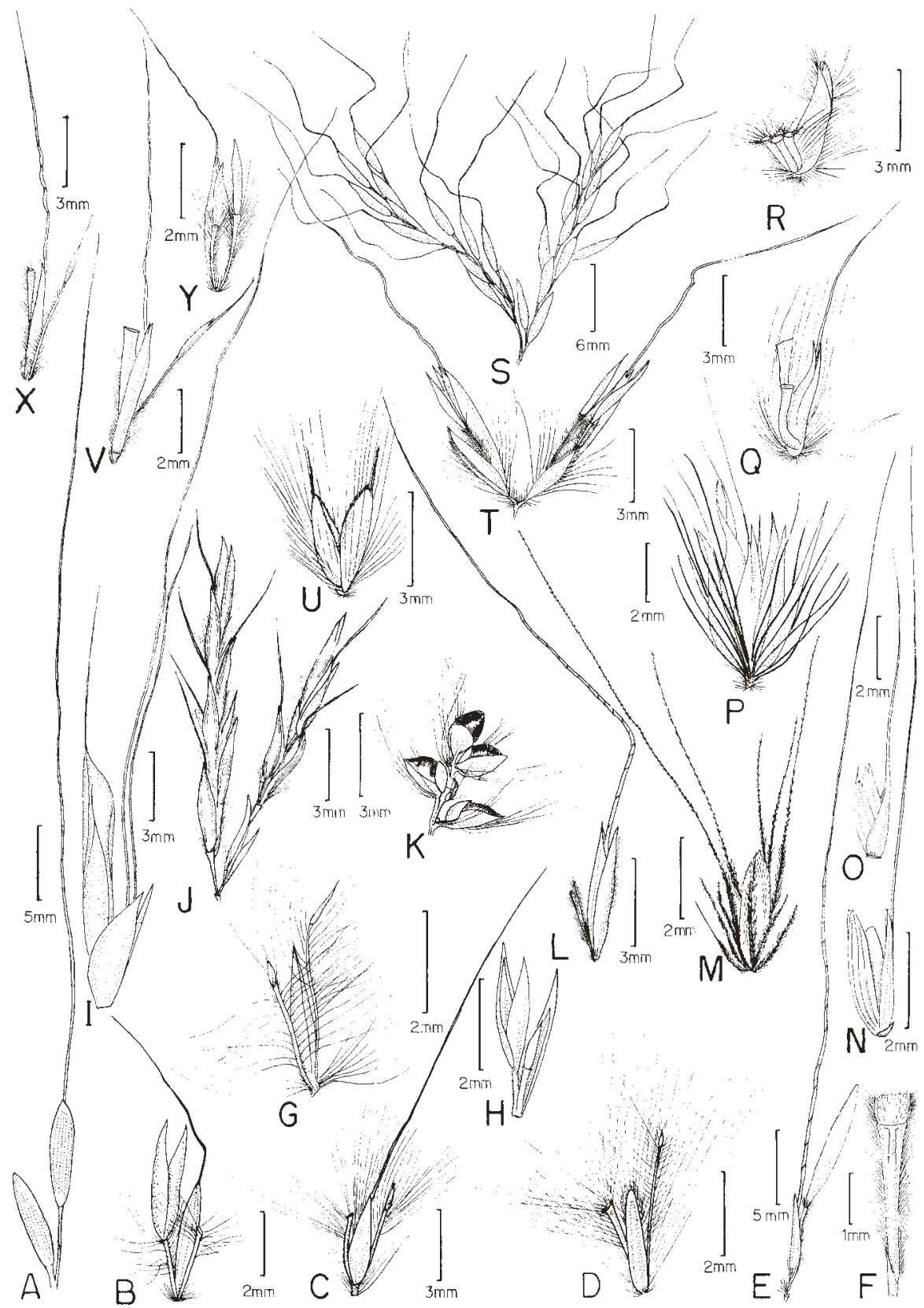

Figura 2. A - Trachypogon spicatus, par de espiguetas; B - Andropogon lateralis, par de espiguetas; C - A. macrothrix, par de espiguetas; D - A. leucostachyus, par de espiguetas; E - Agenium leptocladum, par superior de espiguetas; F detalhe do calo da espigueta séssil; G - Andropogon bicornis, espiguetas apicais do racemo; H - A. virgatus, par de espiguetas; I - A. fastigiatus, par de espiguetas; J - Cymbopogon densiflorus, racemos conjugados; K - Setaria parviflora, corte da inflorescência mostrando espiguetas envoltas por cerdas; L - Sorghastrum minarum, espigueta séssil; M Pennisetum polystachyum, espiguetas envoltas por cerdas basais; N - Melinis minutiflora, espigueta; O - Oplismenus hirtellus, espigueta; P - Pennisetum purpureum, espiguetas envoltas por cerdas basais; Q - Saccharum trinii, espigueta séssil; R - Eriochrysis cayennensis, espigueta séssil; S - Hyparrhenia rufa, racemos conjugados; T - H. bracteata, racemos conjugados; U - Rhynchelytrum repens, espigueta; V - Schizachyrium microstachyum, par de espiguetas; $\mathrm{X}$ - S. tenerum, par de espiguetas; Y - S. cf. sanguineum, par de espiguetas. 


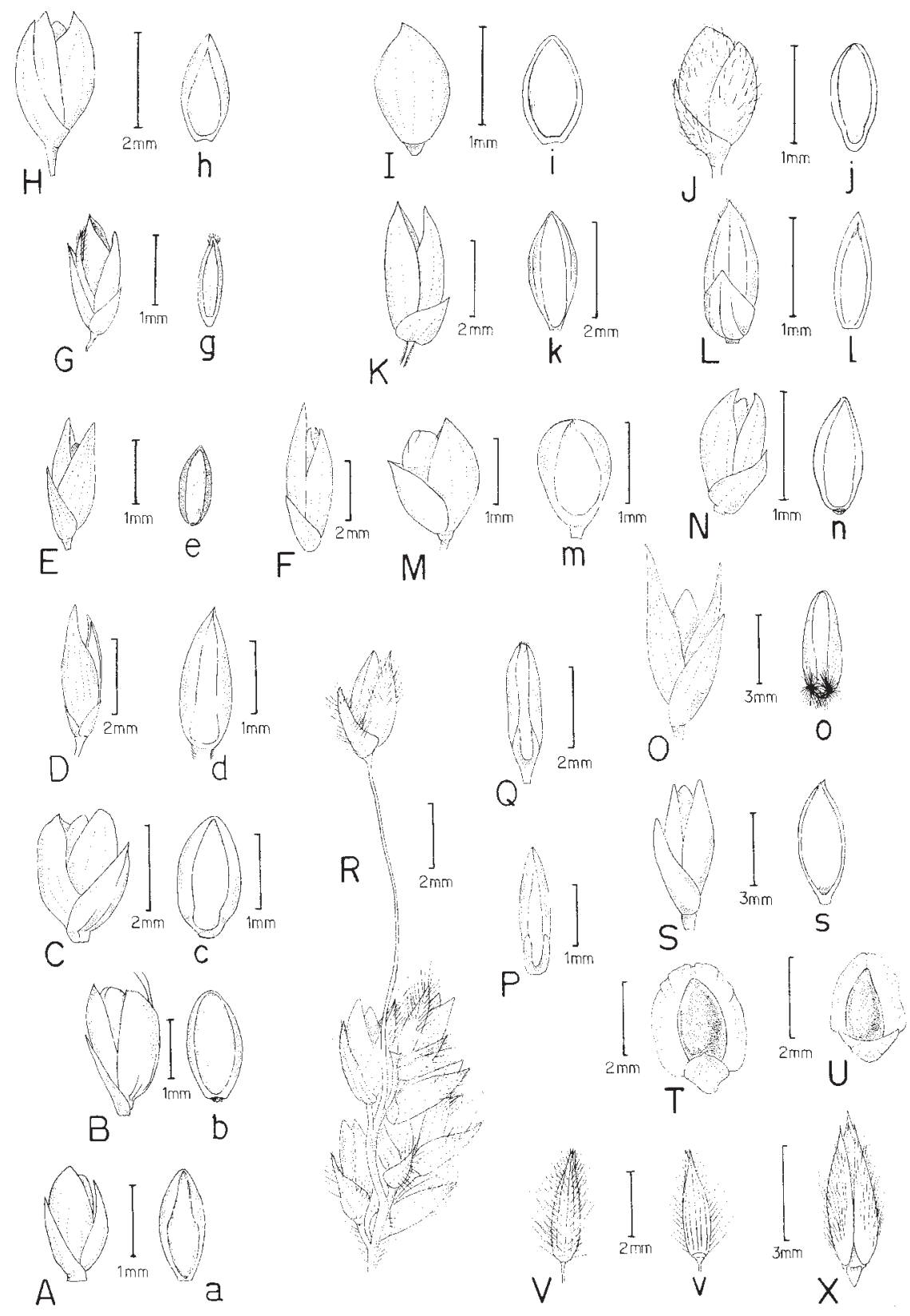

Figura 3. A - Panicum schwackeanum, espigueta, a - flósculo superior; B - P. sellowii, espigueta, b - flósculo superior; $\mathrm{C}$ - P. cyanescens, espigueta, c - flósculo superior; D - P. dichotomiflorum, espigueta, d - flósculo superior; E - P. peladoense, espigueta, e - flósculo superior; F - Steinchisma hians, espigueta; G - S. decipiens, espigueta, g - flósculo superior; H - Panicum caaguazuense, espigueta, h - flósculo superior; I - P. trichanthum, espigueta, i - flósculo superior; J - P. pseudisachne, espigueta, j - flósculo superior; K - P. maximum, espigueta, k - flósculo superior; $\mathrm{L}$ - $P$. laxum, espigueta, 1 - flósculo superior; $\mathrm{M}$ - P. parvifolium, espigueta, $\mathrm{m}$ - flósculo superior; $\mathrm{N}$ - P. pilosum, espigueta, $\mathrm{n}$ - flósculo superior; O - P. olyroides, espigueta, o - flósculo superior; P - Ichnanthus pallens, flósculo superior; Q - I. tenuis, flósculo superior; R - I. procurrens, ramo da inflorescência; S - Acroceras zizanioides, espigueta, s - flósculo superior; T - Otachyrium versicolor, espigueta mostrando alas da pálea inferior; U - O. seminudum, espiguetas mostrando alas da pálea inferior; V - Leptocoryphium lanatum, espigueta vista ventral, v - espigueta vista dorsal; X - Thrasya petrosa, espigueta. 


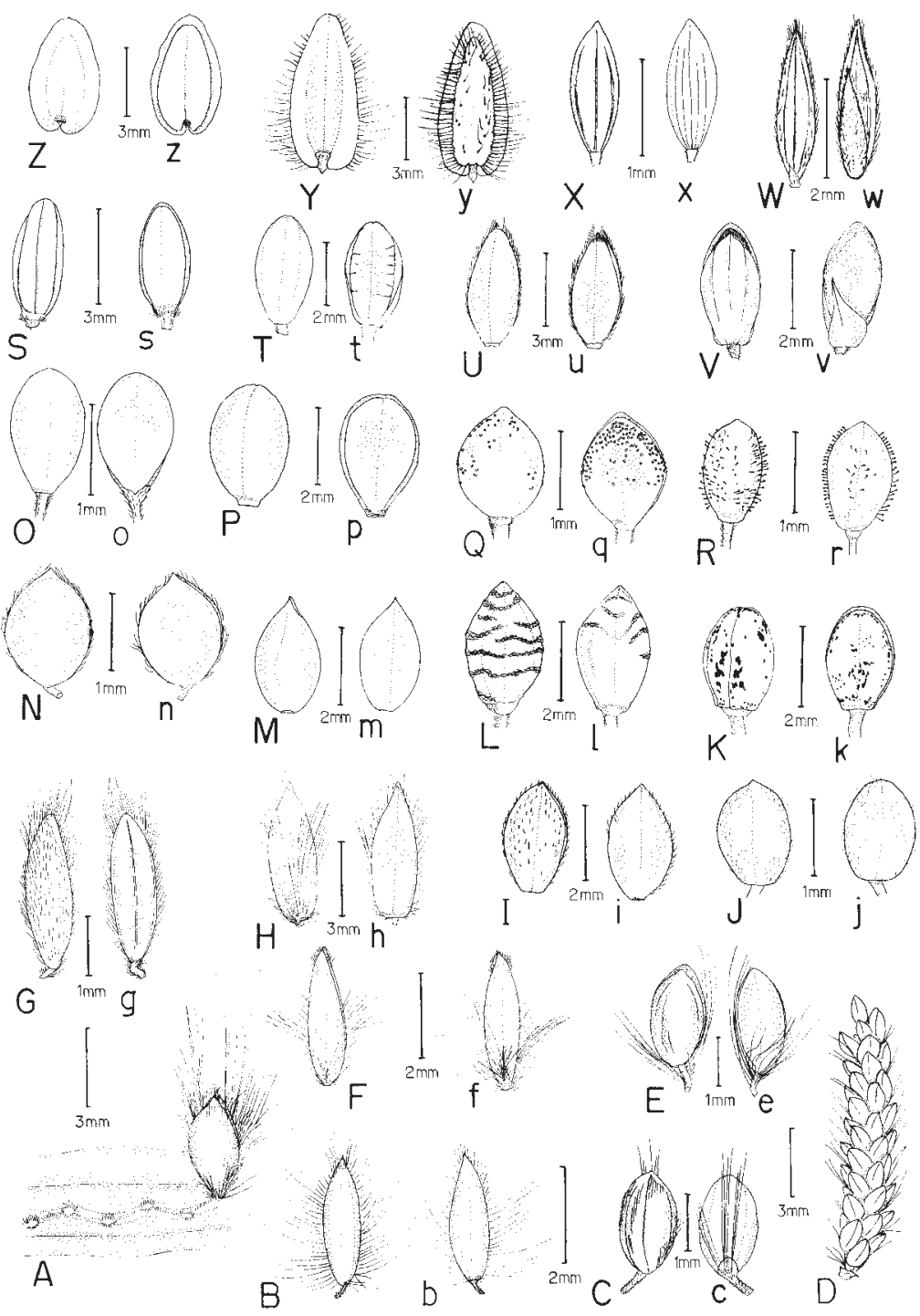

Figura 4. A - Paspalum stellatum, corte do racemo mostrando raque alada e "estrela" de pêlos em torno dos pedicelos; B - P. polyphyllum, espigueta, vista dorsal, b - espigueta, vista ventral; C - P. reduncum, espigueta, vista ventral, c espigueta, vista dorsal, D - racemo mostrando agregação das espiguetas; E - P. gardnerianum, espigueta, vista ventral, e - espigueta, vista dorsal; G - P. trachycoleon, espigueta, vista ventral, g - espigueta, vista dorsal; H - P. erianthum, espigueta, vista ventral, h - espigueta, vista dorsal; I - P. conspersum, espigueta, vista ventral, i - espigueta, vista dorsal; J - P. paniculatum, espigueta, vista ventral, $\mathrm{j}$ - espigueta, vista dorsal; $\mathrm{K}$ - P. maculosum, espigueta, vista ventral, k - espigueta, vista dorsal; L - P. geminiflorum, espigueta, vista ventral, 1 - espigueta, vista dorsal; $\mathrm{M}$ - $P$. fasciculatum, espigueta, vista ventral, $\mathrm{m}$ - espigueta, vista dorsal; $\mathrm{N}$ - P. conjugatum, espigueta, vista ventral, $\mathrm{n}$ espigueta, vista dorsal; O - P. hyalinum, espigueta, vista ventral, o - espigueta, vista dorsal; P - P. plicatulum, espigueta, vista ventral, $\mathrm{p}$ - espigueta, vista dorsal; Q - P. multicaule, espigueta, vista ventral, q - espigueta, vista dorsal; R - $P$. clavuliferum, espigueta, vista ventral, $\mathrm{r}$ - espigueta, vista dorsal; $\mathrm{S}$ - P. dedeccae, espigueta, vista ventral, $\mathrm{s}$ - espigueta, vista dorsal; T - P. glaucescens, espigueta, vista ventral, t - espigueta, vista dorsal; U - P. ellipticum, espigueta, vista ventral, u - espigueta, vista dorsal; V - P. pilosum, espigueta, vista ventral, v - espigueta, vista dorsal; W - Digitaria ciliaris, espigueta, vista dorsal, $\mathrm{w}$ - espigueta, vista ventral; $\mathrm{X}$ - D. violascens, espigueta, vista dorsal, $\mathrm{x}$ - espigueta, vista ventral; Y - Paspalum pectinatum, espigueta, vista ventral, y - espigueta, vista dorsal; Z - P. imbricatum espigueta, vista ventral, $\mathrm{z}$ - espigueta, vista dorsal. 


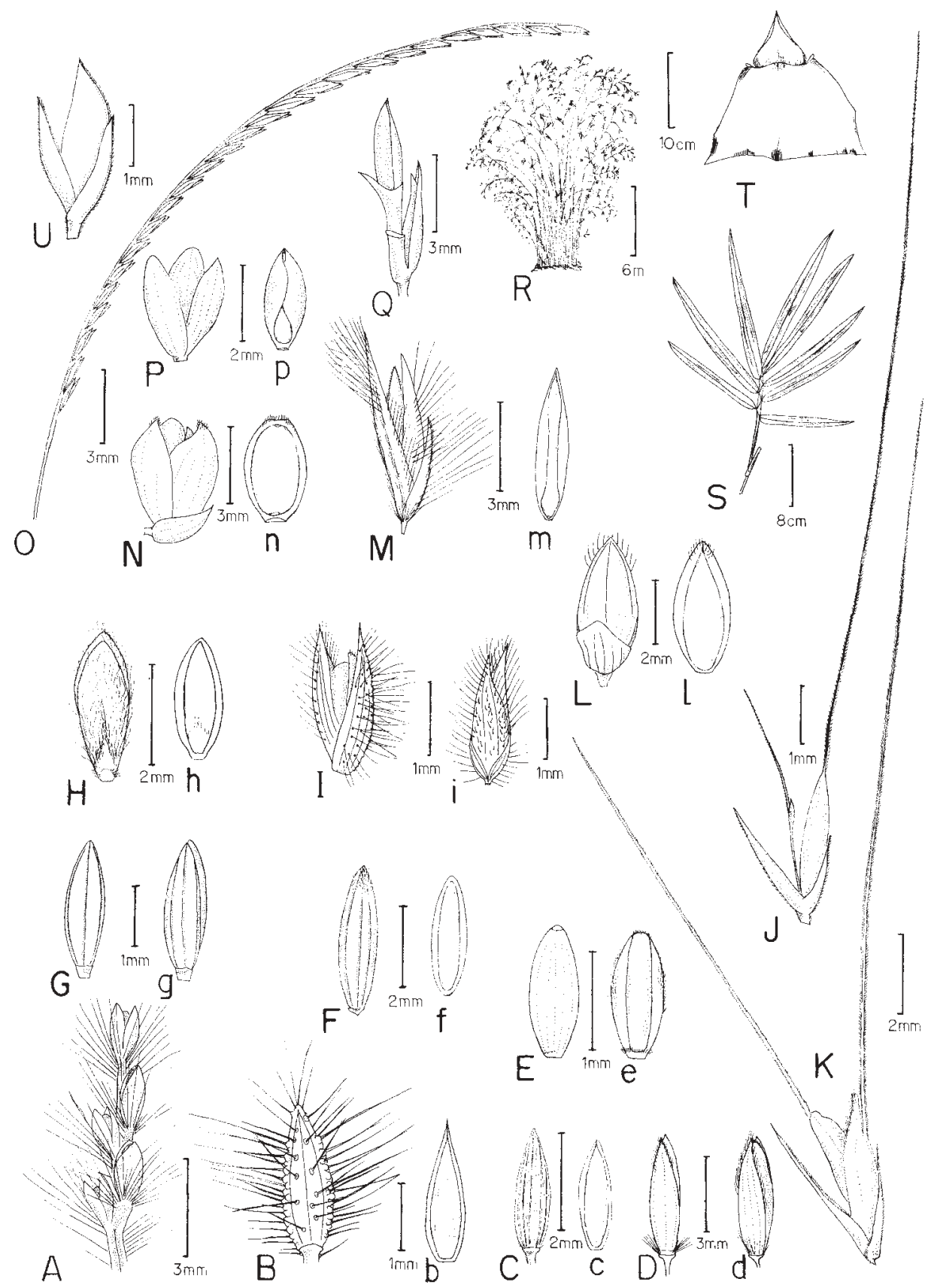

Figura 5. A - Axonopus aureus, corte do racemo mostrando pilosidade na base das espiguetas; B - Axonopus brasiliensis, espigueta, b - flósculo superior; C - Axonopus barbigerus, espigueta, c - flósculo superior; D - Axonopus equitans, espigueta, d - flósculo superior; E - Axonopus capillaris, espigueta, e - flósculo superior; F- Axonopus comans, espigueta, f - flósculo superior; G - Axonopus siccus, espigueta, g - flósculo superior, $\mathrm{H}$ - Axonopus marginatus, espigueta, $\mathrm{h}$ flósculo superior; I - Echinolaena inflexa, espigueta vista lateral, i - espigueta vista ventral.; J - Chloris pycnothrix, espigueta; K - C. orthonoton, espigueta; L - Urochloa brizantha, espigueta, 1 - flósculo superior; M - Mesosetum ferrugineum, espigueta, $\mathrm{m}$ - flósculos superior; $\mathrm{N}$ - Lasiacis ligulata, espigueta, n - flósculo superior; O - Microchloa indica, racemo solitário; P - Homolepsis glutinosa, espigueta, p - flósculo superior; Q - Coelorachis aurita, par de espiguetas; R - Bambusa vulgaris, hábito, S - folha do ramo, T - folha do colmo; U - Cynodon dactylon, espigueta. 


\section{Sporobolus R. Br.}

1. Ramos da inflorescência desprovidos de espiguetas nos $1 / 2$ a 1/5 basais (Fig. 1-R)

S. acuminatus

1. Ramos da inflorescência com espiguetas até na base

2. Plantas anuais, $10-25 \mathrm{~cm}$ compr.; bainhas e lâminas foliares pilosas (Fig.1-Q)

S. ciliatus

2. Plantas perenes, 30-90cm compr.; bainhas e lâminas foliares glabras (Fig.1-P)

S. indicus

\section{Steinchisma Raf.}

1. Panícula espiciforme (Fig. 3-G-g)

S. decipiens

1. Panícula semi-contraída (Fig. 3-F) S. hians

\section{Agradecimentos}

Aos professores e funcionários do Departamento de Botânica da Universidade de Brasília; aos funcionários da Reserva Ecológica do IBGE, Francisco das Chagas Oliveira, Diacis Alvarenga e Maria do Socorro; aos curadores dos herbários consultados: UB, IBGE, CEN e $\mathrm{HEPH}$. Tarciso S. Filgueiras agradece ao $\mathrm{CNPq}$ pela bolsa de produtividade em pesquisa $2 \mathrm{~A}$ (processo 301190/86-0) e ao Departamento de Agronomia da UPIS - Faculdades Integradas, pelo apoio recebido; à Cristina Garcez, pelas ilustrações.

\section{Referências bibliográficas}

Boechat, S. C. 1998. As espécies do gênero Eragrostis Wolf (Poaceae) no Brasil. Tese de Doutorado. Universidade Federal do Rio Grande do Sul, Porto Alegre.

Boechat, S. C. \& Longhi-Wagner, H. M. 1995. O gênero Sporobolus (Poaceae: Chloridoideae) no Brasil. Acta Botanica Brasilica 9(1): 21-86.

Boldrini, I. I. 1976. Gramíneas do gênero Setaria no Rio Grande do Sul. Anuário Técnico do Instituto de Pesquisas Zootécnicas Francisco Osório 3: 331-422.

Burman, A. G. 1985. Nature and composition of the grass flora of Brazil. Willdenowia 15: 211-233.

Chase, A. 1942. The North American Species of Paspalum. Contributions From the United States National Herbarium 28: 1-310.
Clayton, W. D. 1969. A revision of the genus Hyparrhenia. Kew Bulletin, Additional Series II, London.

Clayton, W. D. \& Renvoize, S. A. 1986. Genera Graminum. Royal Botanic Gardens, London.

Filgueiras, T. S. 1982. Taxonomia e distribuição de Arthropogon Nees (Gramineae). (1982). Bradea 3(36): 303-322.

Filgueiras, T. S. 1991. A floristic analisys of the Gramineae of Brazil's Distrito Federal and a list of species occurring in the area. Edinburgh Journal of Botany 48: 73-80.

Filgueiras, T. S. 1992. Gramíneas forrageiras nativas no Distrito Federal, Brasil. Pesquisa Agropecuária Brasileira 27(8): 1103-1111.

Filgueiras, T. S. 1995. Gramineae (Poaceae) In: J. A. Rizzo (coord.). Flora dos Estados de Goiás e Tocantins, Coleção Rizzo, v. 17.

FUNATURA. 1994. Plano de Manejo da Área de Relevante Interesse Ecológico do Riacho Fundo.

Judziewicz, E. 1990. Poaceae. In: A. R. A. Gorts-Van Rijn. Flora of the Guiana. Koeltz Scientific Books, Koenigstein.

Longhi-Wagner, H. M. 1999. O gênero Aristida no Brasil. Boletim do Instituto de Botânica 12: 113-179.

Martins, C. R. 1996. Revegetação com gramíneas de uma área degradada no Parque Nacional de Brasília-DF, Brasil. Dissertação de Mestrado. Universidade de Brasília, Brasília.

Mendonça, R. C.; Felfili, J. M.; Walter, B. M. T.; SilvaJunior, M. C.; Rezende, A. V.; Filgueiras, T. S. \& Nogueira, P. E. 1998. Flora Vascular do Cerrado. Pp. 289-556. In: S. M. Sano \& S. P. Almeida. Cerrado Ambiente e Flora. 
Nicora, E. G. \& Rúgolo de Agrasar, Z. E. 1987. Los géneros de gramíneas de America Austral. Editorial Hemisferio Sur, Buenos Aires.

Pohl, R. W. 1980. Gramineae. In: W. Burger. Flora Costaricensis. Fieldiana: Botany, New Series, 4.

Renvoize, S. A. 1984. The Grasses of Bahia. Royal Botanic Gardens, London.

Rodrigues-da-Silva, R. 2000. Gramíneas (Poaceae) da Área de Relevante Interrese Ecológico (ARIE) Santuário de Vida Silvestre do Riacho Fundo, DF - Brasil. Dissertação de Mestrado. Universidade de Brasília, Brasília.

SEMATEC. 1992. Mapa Ambiental do Distrito Federal. Secretaria do Meio Ambiente, Ciência e Tecnologia do Governo do Distrito Federal, Brasília.

Sendulsky, T. \& Soderstrom, T. R. 1984. Revision of the South American Genus Otachyrium (Poaceae: Panicoideae). Smithsonian Contributions to Botany 57: 1-24.
Smith, L. B. \& Wasshausen, D. C. 1981. Chave para os gêneros de gramíneas brasileiras. Bradea 3: 1-36

Stieber, M. T. 1982. Revision of Ichnanthus sect. Ichnanthus (Gramineae: Panicoideae). Systematic Botany 7(1): 85-115.

Stieber, M. T. 1987. Revision of Ichnanthus sect. Foveolatus (Gramineae: Panicoideae). Systematic Botany 12(2): 187-216.

Watson, L.; Clifford, H. T. \& Dallwitz, M. J. 1985. The classification of the Poaceae: subfamílias and supertribes. Australian Journal of Botany 33: 433-484.

Watson, L. \& Dalwitz, M. J. 1992. The grass genera of the world. C. A. B. International, Wallingford.

Zuloaga, F. O.; Morrone, O.; Rúgulo de Agrasar, Z. E.; Anton, A. M.; Arriaga, M. O. \& Cialdella, A. M. 1994. Flora del Paraguay 23. Gramineae V: 1-326. 